\title{
LAS TELECOMUNICACIONES BASE PARA LA CONFIGURACIÓN DE UN NUEVO ORDEN TERRITORIAL EN ANDALUCÍA
}

Inmaculada ESPAÑA RÍOS*

\section{INTRODUCCIÓN}

El actual proceso de cambio y transformación que hoy vive Andalucía delineado a partir de actuaciones político-económicas, sociales e, incluso, culturales está contribuyendo sin lugar a dudas a revalorizar y potenciar el posicionamiento de la CC.Autónoma de Andalucía frente al Mundo, en tanto abierta al progreso y la modernidad.

Es por esto que en el marco del actual proceso de modernización del sistema productivo, de mejora de la calidad de vida y de formación de recursos humanos, que se impulsa desde Andalucía, el desarrollo del sistema de telecomunicaciones asume una importancia fundamental sobre todo si se tiene presente los múltiples perfiles hacia los que se manifiesta: potenciación del desarrollo socioeconómico, científico-tecnológico e, incluso, cultural.

A tal propósito, es oportuno recordar que el actual proceso de desarrollo socioeconómico propuesto para Andalucía se define por la creciente relevancia cuantitativa y el elevado grado de diversificación cualitativa de la dotación de infraestructuras -véase cuadro I sobre el que se observa la representatividad del capítulo de infraestructuras cifrado en un 5,04\% sobre el total del Presupuesto de la CC.AA. de Andalucía en 1994 y la disminusción puntual de la representatividad del mismo en relación al porcentaje que le correspondió en 1993 explicable en este caso en orden a que en este año se cerró el capítulo de inversión correspondiente a las actuaciones emprendidas en Andalucía con motivo celebración de la Exposición Universal de Sevilla de 1992- y de sus prestaciones, fenómenos éstos que se pueden poner en relación, paralelamente, con la

* Colaboradora Honoraria del Departamento de Geografía Física y Análisis Geográfico Regional. Universidad de Sevilla 
necesidad de subsanar problemas conexos a la concentración de la actividad económica, empresarial y/o laboral entorno a ámbitos territoriales muy concretos, al escaso grado de integración de las economías locales, o, a la progresiva concentración de la población en reducidas áreas urbanas o metropolitanas. Así, se indica en documentos oficiales como son las Bases para la Ordenación del Territorio en Andalucía (1990) o el Plan Andaluz de Desarrollo Económico (19911994).

De este modo, Andalucía, afrontando el reto del desarrollo, parece estar volcando una parte importante de sus esfuerzos en la potenciación de las telecomunicaciones, ya que la agilidad de adaptación de este sector al nuevo marco que se diseña para las telecomunicaciones europeas y/o internacionales será decisiva e influirá en el resto de las actividades y decisiones política-económicas, sociales e, incluso, culturales de la Comunidad.

\section{CUADRO I}

PRESUPUESTOS 1993/1994 POR POLÍTICAS DE GASTO (Miles de ptas.)

\begin{tabular}{lrrrrr}
\hline Política de Gasto & Ppto.1993 & \% s/Tot. & Ppto.1994 & \% s/Tot. & \% Var. \\
\hline Sanidad & 516.707 .867 & 31,44 & 565.049 .638 & 31,38 & 9,36 \\
Educación & 415.082 .344 & 25,25 & 436.495 .609 & 24,24 & 5,16 \\
Atención Social & 92.661 .14 & 15,64 & 98.911 .112 & 5,49 & 6,74 \\
Cultura & 20.074 .242 & 1,22 & 22.766 .461 & 1,26 & 13,41 \\
Vivienda y Urbanismo & 41.045 .002 & 2,50 & 40.898 .448 & 2,27 & $-0,36$ \\
Seguridad y Protec. Civil & 2.121 .865 & 0,13 & 2.074 .893 & 0,12 & $-2,21$ \\
Promoción de Empleo & 33.360 .754 & 2,03 & 46.093 .356 & 2,56 & 38,17 \\
Medio Ambiente & 18.080 .715 & 1,10 & 18.629 .045 & 1,03 & 3,03 \\
Cooperac.Económica y & & & & & \\
Relac.Financ.con CC.LL & 159.124 .073 & 9,68 & 160.016 .713 & 8,89 & 0,56 \\
Infraestructuras & 86.373 .261 & 5,26 & 90.755 .874 & 5,04 & 5,07 \\
Fomento Económico & 29.760 .681 & 1,81 & 32.846 .726 & 1,82 & 10,37 \\
Agricultura y Pesca & 56.740 .174 & 3,45 & 83.848 .892 & 4,66 & 47,78 \\
Investigación & 23.121 .335 & 1,41 & 24.372 .554 & 1,35 & 5,41 \\
Deuda Pública & 89.116 .100 & 5,42 & 123.315 .263 & 6,85 & 38,38 \\
Otras Políticas & 60.264 .665 & 3,67 & 54.428 .906 & 3,02 & $-9,68$ \\
\hline TOTAL POLÍTICAS & 1.643 .634 .219 & 100,00 & 1.800 .503 .490 & 100,00 & 9,54 \\
\hline
\end{tabular}

Fuente: Andalucía Económica, 1994. 
Por ello, tanto la Administración Estatal como la Autonómica Andaluza, siguiendo las mismas directrices políticas de la Unión Europea, han propiciado un conjunto de actuaciones impulsadas directamente tanto desde el Gobierno Central, a través del Ministerio de Obras Públicas y Transportes, como desde la Consejería de Obras Públicas y Transportes de la Junta de Andalucía, que pretenden, inicialmente, incrementar los equipamientos de base de telecomunicaciones, fomentar la oferta/demanda de servicios avanzados de telecomunicaciones, insertar los nuevos productos y las tecnologías aquí desarrolladas en el mercado europeo y/o internacional y, en definitiva, incrementar el número de proyectos de $\mathrm{I}+\mathrm{D}$, para el desarrollo de nuevas tecnologías de telecomunicaciones en la región.

Pese a todo, hoy en Andalucía, al igual que sucede en el resto de las regiones de la Unión Europea, como consecuencia de la internacionalización del sector, el desarrollo de las telecomunicaciones, está viéndose determinado por factores ligados al mercado, disponibilidad de tecnologías y por la dinámica de los procesos de regulación. Más, hoy la conjunción del funcionamiento de estos factores se ve perturbada por la debilidad del mismo aparato económico regional, al interponer límites de relevante importancia para el sector. Enlazando, además, todo este panorama con la actual problemática planteada por la desuniformidad de las condiciones de desarrollo a nivel intrarregional en Andalucía, puede pensarse que, en realidad, el contexto para las telecomunicaciones en esta región se muestra hetereogéneo.

A nivel regional el mantenimiento de desequilibrios socioeconómicos es un hecho real, que ha conducido al desencadenamiento de una supeditación tecnológica, al configurarse áreas con gran capacidad innovadora, donde el desarrollo de las telecomunicaciones está propiciando el avance hacia la ya denominada «sociedad de la información», en contraposición con otras más atrasadas, que claramente muestran desventajas que le imposibilitan para dar el necesario salto tecnológico, empresarial y de mercado. Ello es al menos lo que se desprende de la lectura del cuadro II sobre el que se observa como existe una fuerte disparidad en cuanto a dotación de telecomunicaciones, entre el litoral y el interior, con la excepción evidente de la capital autonómica, Sevilla.

Así, lo planes de la Dirección General de Telecomunicaciones del Ministerio de Obras Públicas y Transportes se han propuesto subsanar las disparidades intrarregionales en lo que afecta a dotación de infraestructuras de telecomunicaciones por medio de las actuaciones emprendidas a partir de la operatividad del Programa STAR en Andalucía y la colaboración de las Diputaciones y Administraciones Locales. Por esto, en la provincia de Sevilla se han puesto en marcha tres proyectos: el ARTIDE, para la utilización de recursos y tecnologías de la información como soporte para el desarrollo económico, las OSIT, Oficinas de 
Servicios Integrados de Telecomunicaciones, y el ALTEX I-II-III, para la implantación y desarrollo del servicio videotex.

\section{CUADRO II}

IMPLANTACIÓN DE INFRAESTRUCTURAS DE

TELECOMUNICACIONES EN ANDALUCÍA, 1989. (\% S/Total Nacional)

\section{Andalucía Sevilla Jaén Málaga Cádiz}

Circuitos Telefónico/Digitales

Circuitos Red Telefónica Conmutada

Capacidad Ibertex

Conexiones Iberpac

Fibra Óptica

Líneas Servicio Ibercom

Datáfonos

Telefonía Móvil Automática

Abonados Telefonía Móvil Automática

14,89

1,74

1,26

14,47

5,12

20,63

2,87

15,38

14,70
4,5

0,5

0,3

3,2

0,1

1,5

0,8

2,9

3,6
0,6

2,8

0,1

0,2

0,3

2,6

0,2

0,1

0,2

2,9

4,2

1,7

0,0

0,0

0,4

0,4

0,1

1,3

16,3

0,6

5,4

0,2

3,1

5,6

2,2

Fuente: Estudio Mesa STAR, IFA y FUNDESCO. 1990.

En R.Jordá, 1992.

Con ello los propósitos de la Dirección General de Telecomunicaciones pretende hacer extensivas estas actuaciones a todas las provincias andaluzas, concentrando las dotaciones de infraestructuras en aquellos núcleos de población que desarrollen alguna actividad económica de carácter industrial y/o servicios, con el objeto de potenciar las cabeceras comarcales y vertebrar el territorio regional. En esta misma línea, la Dirección General de Telecomunicaciones y Telefónica de España S.A. han puesto en marcha un Plan Piloto de Telefonía Rural mediante Acceso Celular, que en el caso de Andalucía es operativo, desde abril de 1992, en las provincias de Granada y Almería, con el objeto de garantizar la cobertura de comunicaciones en zonas secularmente aisladas.

Frente a toda esta problemática inicial se entiende, que el objetivo fundamental de este estudio se proponga definir el orden territorial que diseña el actual sistema de telecomunicaciones en Andalucía, por su incidencia y contribución al desarrollo mismo de la región.

Para ello, se pretende observar si tal y como a priori puede pensarse, desde un punto de vista territorial, la mejora del sistema de telecomunicaciones se polariza 
entorno a las grandes ciudades, tal y como es el caso de Sevilla y Málaga, y en menor medida sobre aquellos centros que mantienen niveles estables de demanda de telecomunicaciones, puesto que la modernización del sistema se impone en orden al incremento real y/o previsible de la demanda, y se articula en base al modelo territorial que se pretende consolidar en Andalucía, concretado a partir de documentos como Sistema de Ciudades (1985) y Bases para la Ordenación del Territorio en Andalucía (1990).

A partir de lo expuesto hasta el momento, debe entenderse que la perspectiva adoptada para el desarrollo de este estudio sea globalizadora, en el sentido de que se vislumbra necesario observar las líneas rectoras que dirigen el comportamiento de este sector tecnológico, sin olvidar que aquí serán valoradas desde la óptica del análisis geográfico, ante el intento último de manifestar su incidencia sobre la organización y/u ordenación territorial de Andalucía.

Desde esta perspectiva, es necesario tener presente cómo ha sido el desarrollo de las telecomunicaciones el que tímidamente ha conseguido y conseguirá en un futuro superar las limitaciones que en otro tiempo imponía el factor distancia. Elemento éste determinante del carácter de las relaciones socioeconómicas en la CC.AA. de Andalucía, al constituirse en medio adecuado para el desarrollo de un espacio de flujos de comunicación, que revalorice la estratégica localización regional, como se sabe puente entre Europa y África y eje para las comunicaciones entre el Mediterráneo y el Atlántico; e, integre a nivel interno y externo el territorio andaluz en el espacio socioeconómico nacional, europeo e intemacional.

\section{LA CONSOLIDACIÓN DE UN NUEVO ORDEN TERRITORIAL EN LA CC.AA. ANDALUZA}

Las pautas que establecen las directrices de política territorial en Andalucía inciden claramente en la necesidad de articular internamente el territorio regional, como objetivo base a la hora de plantear cualquier tipo de intervención ya sea sectorial y/o territorial. Por ello, las actuaciones que se proponen en materia de comunicaciones se plantean a partir de las prescripciones que establece el mismo modelo de ordenación territorial propuesto por la Administración Autonómica Andaluza, concretado partir de aquel primer documento elaborado por la Dirección General de Ordenación del Territorio de la Consejería de Política Territorial de la Junta de Andalucía que fue Sistemas de Ciudades (1985), en base al cual la Consejería de Obras Públicas y Transportes elaboró años más tarde las Bases para la Ordenación del Territorio en Andalucía (1990).

Y es que el Estatuto de Autonomía para Andalucía establece de acuerdo con 
la Constitución Española (art.148.3 $3^{\prime}$ ) la competencia exclusiva de la Comunidad Autónoma en política territorial y ordenación del territorio, mientras el Estado se reserva expresamente las competencias en materia de comunicaciones (art.149.21 $\mathrm{CE}^{2}$ ), circunstancia que ha conllevado paralelamente que el diseño de la política general de infraestructuras propuesta por el Estado se adecúe, en la práctica, al modelo territorial que ha diseñado la CC.AA. en desarrollo de sus competencias.

Dentro de este marco base, conviene precisar las circunstancias que se imponen para con el desarrollo de un sistema base de telecomunicación en Andalucía, dado que si bien es opinión comúnmente aceptada que efectivamente las telecomunicaciones se convierten hoy en instrumentos de desarrollo económico-social, también es cierto que el actual proceso de liberalización y globalización del sector abre un panorama incierto para el desarrollo futuro de las telecomunicaciones en la región, sobre todo en estos momentos en que por acuerdo del Consejo (16/6/1993) la liberalización de los servicios de telecomunicaciones se ha hecho extensiva a los servicios públicos de telefonía vocal.

Este hecho deja al descubierto claramente las disfuncionalidades que interpone la apertura plena de las telecomunicaciones a este régimen de libre competencia, más aún en una región como Andalucía en la que los desequilibrios socieconómicos podrán verse incrementados dado que en un futuro no muy lejano cualquier operador podrá introducirse y ofertar sus servicios en el mercado que desee, posiblemente aquel que le resulte más rentable.

Es por esto que se partirá en el desarrollo de este punto del análisis de la realidad de socioeconómica andaluza para, a partir de ella, esbozar el modelo territorial que se describe para la región con el objetivo último de observar las modificaciones que introduce sobre éste el diseño del sistema de telecomunicaciones en Andalucía.

\subsection{El nivel de desarrollo socioeconómico andaluz y la constatación de desequilibrios socioeconómicos a nivel intrarregional.}

Incrementar el nivel de desarrollo socieconómico que mantiene Andalucía es sin duda el objetivo de la actual política económica andaluza tal y como indica el mismo Plan de Desarollo Económico Andaluz (1990-1994), sin embargo son múlti-

1. Art. 148.1.3 CE: 1. "Las Comunidades Autónomas podrán asumir competencias en las siguientes materias: $3^{\circ}$ Ordenación del Territorio, urbanismo y vivienda".

2. Art, $149.1 .21^{\circ} \mathrm{CE}: 1$. "El Estado tiene competencia exclusiva sobre las siguientes materias: $21^{\circ}$ Ferrocarriles y transportes terrestres que transcurran por el territorio de más de una Comunidad Autónoma; régimen general de comunicaciones; tráfico y circulación de vehículos a motor; correos y telecomunicaciones; cables aéreos, submarinos y radio comunicación". 
ples los problemas que aquejan e interponen limitaciones de base a la región justificando el mantenimiento del bajo nivel de desarrollo socioeconómico que la región manifiesta frente al conjunto tanto europeo como nacional -véase cuadro III-.

En este sentido, el retraso económico que manifiesta Andalucía por comparación al nivel de desarrollo de las restantes regiones españolas, puede pensarse en opinión de la Dra.R.Jordá tiene origen en la aplicación de un modelo de desarrollo económico que tiene escasa capacidad de generar empleo y elevar el nivel de bienestar social, a la vez que en la política económica y regional llevada a cabo en un pasado próximo, dado que ésta fue una de las regiones españolas que menos se benefició de la expansión económica que experimentó España a partir de la década de los 60 . A nivel territorial ésta se tradujo en la expansión de la actividad económica en determinadas áreas como resultado del desarrollo unas veces de la actividad industrial y otras del turismo y la agricultura, sectores protagonistas de la transformación en tanto apoyados por las actuaciones del sector público.

\section{CUADRO III \\ VALOR AÑADIDO BRUTO EN ANDALUCÍA, 1989.}

\begin{tabular}{lrr}
\hline & $\begin{array}{r}\text { Andalucía } \\
\%\end{array}$ & $\begin{array}{r}\text { España } \\
\%\end{array}$ \\
\hline Almería & 6,9 & 0,9 \\
Cádiz & 15,1 & 1,9 \\
Córdoba & 10,2 & 1,3 \\
Granada & 9,8 & 1,2 \\
Huelva & 6,9 & 0,9 \\
Jaén & 8,1 & 1,0 \\
Málaga & 18,6 & 2,4 \\
Sevilla & 24,3 & 3,1 \\
& & \\
ANDALUCÍA & 100,0 & 12,6 \\
\hline ANDALUCÍA (Mill.Ptas.) & & 5.800 .700 \\
ESPAÑA (Mill.Ptas.) & & 45.946 .100 \\
\hline
\end{tabular}

Fuente: Banco Bilbao Vizcaya, 1990.

La aplicación de este modelo de desarrollo conllevó, paralelamente, que ya durante el periodo 1960-75 el sistema de ciudades y de asentamientos rurales andaluces sufrieran modificaciones importantes al acentuarse la tendencia a la concentración de la actividad económica y de la población en el litoral mediterrá- 
neo y en el triángulo Sevilla-Huelva-Cádiz, con el consiguiente abandono del campo como consecuencia de la atracción ejercida por las zonas dinámicas andaluzas que desencadenaron un fuerte desequilibrio en la distribución de la población -véase cuadro IV- y agudizaron el proceso de ruralización de $S^{\text {a }}$ Morena, Cordilleras Béticas y surco Intrabético -a excepción de la vega de Granada- y de amplias zonas del Valle del Guadalquivir.

En la actualidad, esta tendencia se ha visto incrementada como consecuencia de la internacionalización y generalización de la economía de mercado al imponer la integración efectiva de los diferentes núcleos para facilitar así los efectos de difusión y crecimiento de la actividad económica. Se entiende así que aquellos espacios que presenten problemas de desarticulación y/o desconexión sean postergados del desarrollo.

El mismo cuadro $V$ es indicativo de lo que se dice a la vez que revela la relación directa que se produce entre concentración de la población y de la actividad económica ${ }^{3}$. Así, en el caso de la renta generada por kilómetro cuadrado, sólo tres de las provincias andaluzas -Cádiz, Málaga y Sevilla- están muy por encima de la media tanto regional como nacional, duplicando ésta en el caso específico de Málaga la media andaluza -la cual a su vez tan sólo alcanza tres cuartos de la media nacional-. Por contra, las otras cinco provincias presentan índices más o menos similares, por debajo todos ellos de la media nacional, y con Jaén prácticamente en la mitad de la media regional.

No obstante, tal y como evidencia el reciente estudio realizado por R.Esteve (1993) cuando la comparación se efectúa en términos por habitante la situación es bastante más homogénea lo que indica claramente que la distribución de Ia población en el espacio se ve claramente afectada por la actividad económica. Dicho de otra manera, lo que se evidencia es que la generación de la producción es muy distinta según el territorio, por ello las desigualdades espaciales en la generación de valor añadido tienden a acrecentarse, en tanto potenciados por los crecientes desequilibrios demográficos.

Es por esto, que la superación del aislamiento producido en unos casos por motivos meramente físicos -barreras montañosas-, en otros por la desconexión funcional de áreas que propicia la inexistencia de una infraestructura base de comunicaciones y, finalmente, el vacío demográfico generado sobre determinadas áreas en Andalucía plantea un gran reto a la política territorial y las correspondientes políticas sectoriales que habrán de aunar esfuerzos en la tarea de homogeneizar las condiciones de desarrollo en Andalucía. 


\section{CUADRO IV \\ DENSIDADES DE POBLACIÓN DE DERECHO, 1990.(Hab/km )}

\begin{tabular}{lrrrr}
\hline & Provincia & Litoral & $\begin{array}{r}\text { Provincia sin } \\
\text { capital }\end{array}$ & $\begin{array}{r}\text { Provincia sin } \\
\text { litoral }\end{array}$ \\
\hline Almería & 53 & 139 & 35 & 24 \\
Cádiz & 148 & 314 & 126 & 70 \\
Córdoba & 56 & - & 34 & 34 \\
Granada & 65 & 202 & 44 & 60 \\
Huelva & 45 & 145 & 31 & 25 \\
Jaén & 49 & - & 41 & 41 \\
Málaga & 168 & 662 & 91 & 55 \\
Sevilla & 115 & - & 67 & 67 \\
Prov. Costeras & 88 & 283 & 59 & 47 \\
\hline ANDALUCíA & 81 & 283 & 54 & 61 \\
\hline
\end{tabular}

Fuente: Elaboración propia a partir de datos del INE.

CUADRO V

DISTRIBUCIÓN DE VAB POR SUPERFICIE Y HABITANTE, 1985

\begin{tabular}{|c|c|c|c|c|c|c|}
\hline & \multicolumn{3}{|c|}{$V A B / \mathrm{km}$} & \multicolumn{3}{|c|}{ VAB/Cápito } \\
\hline & $\begin{array}{c}\text { Miles } \\
\text { Ptas. }\end{array}$ & $\begin{array}{r}\text { Andalucía } \\
\%\end{array}$ & $\begin{array}{r}\text { España } \\
\%\end{array}$ & $\begin{array}{c}\text { Miles } \\
\text { Ptas. }\end{array}$ & $\begin{array}{r}\text { Andalucía } \\
\%\end{array}$ & $\begin{array}{r}\text { España } \\
\%\end{array}$ \\
\hline Almería & 45.541 & 68,4 & 49,9 & 850 & 104 & 74 \\
\hline Cádiz & 118.891 & 178,9 & 130,6 & 805 & 99 & 70 \\
\hline Córdoba & 43.221 & 65,0 & 47,5 & 770 & 94 & 67 \\
\hline Granada & 45.372 & 68,3 & 49,8 & 696 & 85 & 60 \\
\hline Huelva & 39.757 & 59,8 & 43,7 & 888 & 109 & 77 \\
\hline Jaén & 34.765 & 52,3 & 38,2 & 709 & 87 & 62 \\
\hline Málaga & 148.520 & 223,4 & 163,2 & 883 & 108 & 77 \\
\hline Sevilla & 100.821 & 151,7 & 110,8 & 873 & 107 & 76 \\
\hline ANDALUCÍA & 66.470 & 100,0 & 73,0 & 817 & 100 & 71 \\
\hline ESPAÑA & 91.022 & & 100,0 & 1.152 & & 100 \\
\hline
\end{tabular}

Fuente: Banco de Bilbao Vizcaya, Renta Nacional de España y su distribución provincial, 1987. En M.Martín, 1993. 
Con esta idea entronca de lleno la posibilidad que abren hoy las telecomunicaciones en la tarea de contribuir por un lado al desarrollo de determinados sectores productivos en Andalucía y, por otro, configurar a la región como un espacio de flujos de comunicación.

\subsection{La articulación del territorio andaluz a partir del modelo diseñado por el sistema de ciudades}

Como bien concretó la Consejería de Política Territorial de la Junta de Andalucía en el documento Sistema de Ciudades (1985) el sistema básico de ciudades y sus correspondientes áreas de influencia definen hoy junto a los principales ejes de comunicación el esquema de vertebración territorial de Andalucía.

En él Sevilla, pese a constituirse en centro aún en proceso de consolidación emerge como «primer centro» a escala regional en base a las funciones que asume y relaciones que mantiene a nivel extraprovincial. Más, junto con él son las capitales provinciales, como vertebradoras de los subsistemas urbanos regionales, las que ponen base a la actual estructura territorial andaluza.

Dicha estructura como mantiene J.M $M^{a}$ Feria (1987) no permite identificar un sistema organizado e integrado en la región, debido básicamente a la desarticulación económica de la misma, la propia configuración territorial y geográfica andaluza y la similitud de los tamaños de los mayores asentamientos andaluces.

Por ello, la realidad del sistema urbano muestra igualmente el desequilibrio del sistema de asentamientos, que en su evolución reciente ha favorecido el crecimiento de las grandes ciudades, expansión del litoral, y, la progresiva venida a menos y despoblamiento de las agrociudades, con el consiguiente empobrecimiento del sistema urbano del interior de la región.

Sin embargo, a partir de la interpretación que realiza $M^{\mathrm{a}}$ del C. Ocaña (1993) en relación a la lectura del documento base antes reseñado característica fundamental de la articulación funcional de la región resulta ser la referente a las grandes diferencias en los niveles de articulación a nivel intraprovincial, donde observa coexisten situaciones contrastadas tal y como es el caso de centros funcionalmente importantes que no ejercen centralidad sobre los espacios circundantes -tal es el caso de las campiñas- debido a la homogeneidad de los asentamientos y sus similares bases productivas; áreas igualmente desarticuladas, por la inexistencia de algún núcleo de potencia funcional supramunicipal, como ocurre en gran parte de $\mathrm{S}^{\mathrm{a}}$ Morena y en amplios sectores de las Béticas, con una red muy débil de asentamientos.

Otros, por el contrario, con un sistema de poblamiento débil aparecen articuladas a nivel supramunicipal por pequeñas cabeceras comarcales, normalmente con muy 
escasa potencialidad funcional, hecho frecuente en los valles intramontano: penibéticos y que también existe en la $S^{\mathbf{a}}$ de Aracena, espacios muy alejados de los centros urbanos de mayor entidad. Mejor articulado aparece en general el Valle de Guadalquivir, el Subbético Occidental, el surco Intrabético y el litoral, con una rec de asentamientos equilibrada y núcleos de importante capacidad funcional, que ad. quieren por la densidad y continuidad de la ocupación rasgos de regiones urbanas er los entornos de Sevilla, Granada, Jaén, Cádiz, Algeciras, Málaga, Almería y Huelva.

Actuando sobre esta realidad se propone una jerarquía de articulaciones, quє tiene como consecuencia una clara potenciación de las relaciones a nive organizativo subregional y que describe los siguientes niveles (véase figura 1): e de ámbito regional, los de ámbito subregionales -esencialmente provinciales- y los ámbitos intermedios y básicos como dos jerarquías dentro de las articulacio. nes supramunicipales.

En síntesis, puede decirse cómo en base a esta jerarquía, la función regiona de Sevilla queda en parte limitada tan sólo por su localización y por la importancia de Granada y Málaga, mientras la articulación más definida del espacic andaluz corresponde a las capitales provinciales, con las particularidades de Јетеz y Algeciras, que realizan funciones subregionales y se localizan de forma centra] respecto a la mayoría de municipios.

También, dentro del nivel básico supramunicipal, que es generalizado en las áreas de Campiña, Valle del Guadalquivir, Litoral y Depresiones Penibéticas, se identifican dos jerarquías o niveles -superior e intermedio- que reducen las depencias con relación a las capitales provinciales, si bien es difícil distinguir con nitidez las funciones específicas y el grado de centralidad de cada uno. Son los casos de los centros superiores de Lucena y Pozoblanco en Córdoba; Baza. Guadix y Motril en Granada; Aracena en Huelva; Úbeda, Linares y Andújar en Jaén; y, Antequera, Velez-Málaga y Ronda en Málaga.

A partir de este esquema base la relación entre sistema urbano y de comunicaciones en términos de intervención y por tanto de planificación de actuaciones se concreta en mejorar la accesibilidad entre entre los centros básicos y entre éstos y los superiores, y potenciar los ejes de comunicación que faciliten el intercambic económico intra y extrarregional.

En base a ello, la adecuación del sistema de telecomunicaciones que se propone para Ándalucía al modelo territorial esbozado se articula sobre las premisas que establece la política de infraestructuras, la cual a pesar de plantearse a nivel nacional, acoge en sus iniciativas de intervención las indicaciones que establece a nivel territorial las pautas de la política territorial andaluza.

Sin embargo, se quiere intencionadamente incidir en la importancia estratégica que hoy posee el desarrollo de las infraestructuras de telecomunicaciones en 


\section{FIGURA 1 \\ SISTEMA DE CIUDADES}

Propuesta de la Consejería de Obras Públicas y Transportes

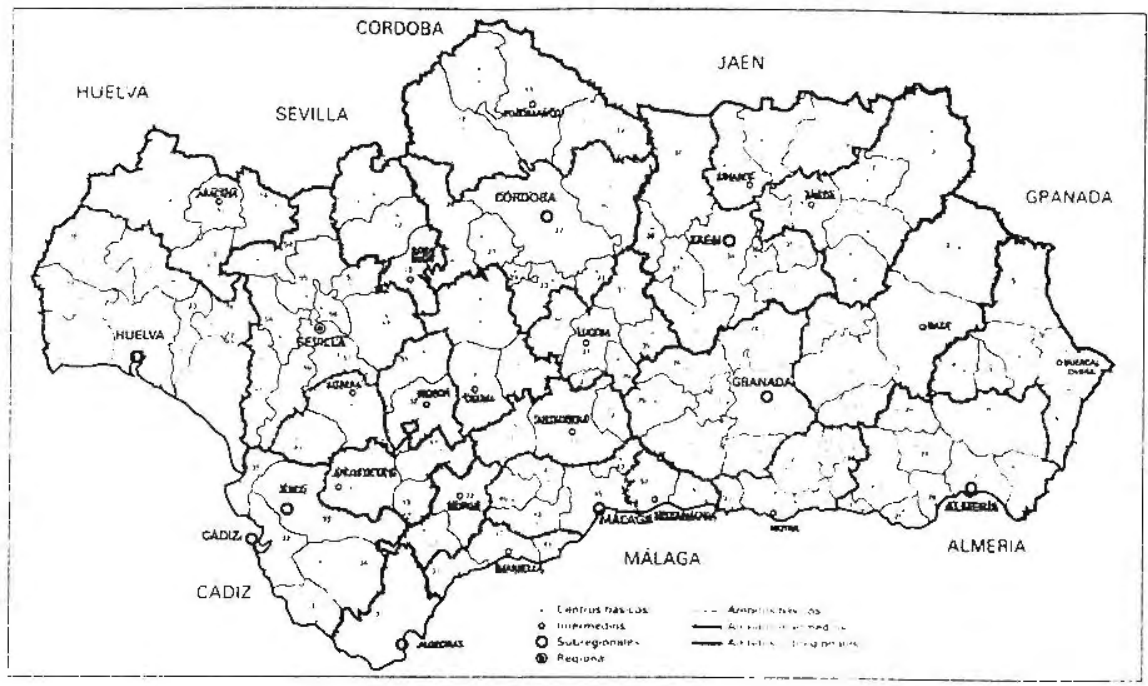

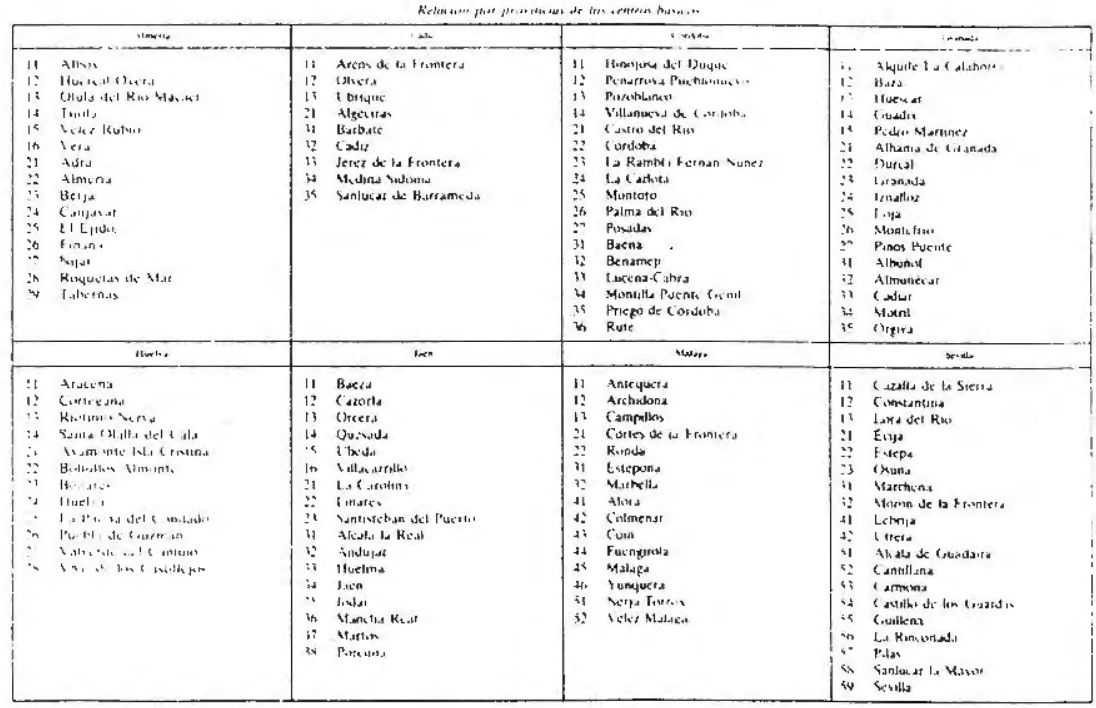

Fuente: Consejería de Política Territorial. Junta de Andalucía, 1985. 
Andalucía, dado que en la sociedad contemporánea el acceso a la información ، intercambio de la misma se constituye en elemento base para el funcionamientc del sistema económico y desarollo de la vida cotidiana.

Desde esta perspectiva, las telecomunicaciones se proponen como infraestruc. tura que cuando se desarrolla y es operativa consigue superar las limitacione: físicas que interpone la distancia o dificultades de comunicación por la inexisten. cia de dotaciones de infraestructuras entre áreas distantes, circunstancias éstas que tradicionalmente han sido y son reseñadas por la literatura comc condicionantes del subdesarrollo andaluz. La transcendencia e importancia de ta hecho exige que a continuación se pase a considerar la articulación del sistema de telecomunicaciones en Andalucía.

\section{LA ACTUALIDAD DEL SISTEMA DE TELECOMUNICACIONES EN ANDALUCÍA}

En los últimos años, se está produciendo un cambio importante en la estructura de las telecomunicaciones en Europa como consecuencia de la velocidad a que se suceden los cambios tecnológicos en el sector, siendo ello, en definitiva, lo que está exigiendo que se implante un nuevo sistema de telecomunicaciones avanzadas en Andalucía, que convierta verdaderamente a la región en un espacio difuso sobre el cual puedan desarrollarse e intensificarse flujos de comunicación.

Por ello, hoy son las modernas tecnologías de fibra óptica, las comunicaciones por satélite y los sistemas digitales las que están permitiendo incrementar la capacidad de transmisión al integrar en una misma red múltiples servicios de telecomunicación.

Sin embargo, para que estas modemas tecnologías puedan ejercer su función económica y social es necesario potenciar el desarrollo de los sistemas soportados por ellas. De aquí, en palabras de E.Zurutuza «surge la importancia estratégica de las redes de cable para las comunicaciones», en tanto su distribución espacial plantea la superación de las limitaciones que hasta el momento en Andalucía venía interponiendo el factor distancia o el aislamiento geográfico como condicionantes de base para el desarrollo integral de la región.

Es por eso que dentro del marco de la CC.AA. de Andalucía, la inversión en este nuevo sistema de telecomunicaciones se convierte en apuesta de futuro. Ello es al menos lo que puede pensarse en función de lo que se observa en el capítulo de inversiones correspondiente a las Memorias de Telefónica, valorables conjun- 
tamente por su elevada cuantía al elevarse a más de 100.000 millones de pesetas, para el periodo 1992-95.

Ciertamente, los propósitos de la Administración Autonómica Andaluza pretenden, a través de este fuerte plan de inversiones, vertebrar internamente el territorio de la CC.AA. y aproximar Andalucía al resto del Mundo y de España, dotándola para ello de modernas infraestructuras y servicios telemáticos, que se materializan en la implantación de una red avanzada fundamentada esencialmente sobre cables de fibra óptica y coaxial, colegados con nodos que enlazan con centrales de clasificación y selección, antenas parabólicas o edificios inteligentes. A partir de las cuales superar las deficiencias que imponen los mismos condicionantes físicos-geográficos, político-económicos y socioculturales que han justificado el tradicional aislamiento y retraso de Andalucía.

En base a ello, las actuaciones que Telefónica de España,S.A. está acometiendo en Andalucía entre 1992-1995 se traducirán, tal y como informaba el mismo Cándido Velázquez (1992) -Presidente de la Entidad-, en que:

- El número de líneas en servicio se elevará desde 1.865 .000 en 1992 hasta 2.300 .000 en 1995 , creciendo en un $23,3 \%$.

- El grado de digitalización, máximo exponente de la modernización de la red, pasará de un 38,7\% en 1992 a un 53,5\% en 1995 .

- La inversión total de Telefónica en la CC.AA. se mantendrá en un nivel algo inferior al máximo, alcanzando en 1990, que llegó a los 90.000 millones.

- Los servicios Ibercom crecerán a un ritmo superior al $30 \%$ acumulativo anual y los de la red Iberpac pasarán de 12.700 en 1992 a 18.000 en 1995.

- La capacidad de oferta de circuitos de la red Ibermic aumentará desde 9.000 hasta 13.000 .

- La Telefonía Móvil Automática, en cuanto a capacidad de los usuarios, se elevará en más del $60 \%$ anual acumulativo en el próximo trienio.

Sin embargo, todas estas iniciativas deben ser consideradas con cautela ya que si bien están contribuyendo, tal y como es el objetivo de Telefónica, a la modemización global del sistema de telecomunicación regional; no debe olvidarse que, por el momento, la desuniformidad de las condiciones de desarrollo a nivel intrarregional está interponiendo condicionantes de base para su homogenea extensión al conjunto de territorio andaluz. Y es que Andalucía cuenta aún con zonas secularmente marginadas, que a causa de la incomunicación social y el aislamiento geográfico se encuentran deabastecidas de servicios base de telecomunicación. 
Por ello, la Dirección General de Telecomunicaciones y Telefónica dt España,S.A. puso en marcha el plan piloto de Telefonía Rural mediante Accesc Celular (TRAC), que en el caso de Andalucía es operativo, desde abril de 1992 en las provincias de Granada y Almería; y, por medio del cual los nuevos abona. dos, de estas zonas desabastecidas, pueden acceder desde su domicilio a las más avanzadas tecnologías en materia de comunicación telefónica, con sólo intalar er su domicilio un equipo radio eléctrico con adaptador (véase figura 2) que s€ conecte a un terminal convencional.

En este contexto, se entiende que el objetivo fundamental propuesto por los operadores de telecomunicaciones, ante la máxima de rentabilizar inversiones, nc sea otro más que aquel de planificar una equilibrada utilización de las tecnologías disponibles, para impulsar así el actual proceso de modernización del sistema de telecomunicaciones andaluz, e, indirectamente, poder llegar a satisfacer las exigencias de los usuarios, a través del incremento de la calidad y amplia difusiór de servicios que se ofrecen.

Más, la consecución de este objetivo ha conllevado el que los mismos operadores mantengan hoy su interés con vistas al desarrollo de la tecnología de los sistemas de telecomunicaciones vía satélite, en tanto que pueden constituirse en un soporte complementario e integrado de los medios terrestres, con funciones que pueden ser articuladas y modeladas en relación a áreas de aplicaciones muy concretas y a la evolución de las exigencias de las redes y de los servicios mismos.

Pese a todo, desde una perspectiva técnico-económica hay que considerar cómo la utilización de los sistemas de transmisión vía satélite no se sustenta, como consecuencia de las cuantiosas inversiones que requieren, sobre la base de la competitividad, sino sobre la máxima de dar validez al objetivo de calidad global del sistema de telecomunicaciones, que hoy persigue el mismo Ministerio de Obras Públicas y Transportes. Desde esta óptica, Telefónica de España, S.A. en colaboración con Retevisión, para hacer frente de forma rápida y adecuada a las exigencias expresas o potenciales de la demanda, tiene en curso diversas realizaciones basadas en el empleo de los sistemas de transmisión instalados a bordo del satélite EUTELSAT e INTELSAT; pero sin duda la empresa más ambiciosa de cuantas a acometido España en el ámbito de las telecomunicaciones vía satélite ha sido la abierta por el satélite experimental HISPASAT, cuya principal misión será la de crear un sistema espacial de telecomunicaciones que sea plenamente operativo hacia 1995.

También, en Andalucía las realizaciones impulsadas en Andalucía con motivo de la celebración de la Exposición Universal de la Exposición Universal de Sevilla de 1992 -Central RDSI, Estación Base de Telefonía Móvil, Centro de 
Control de Videoconferencia, Complejo de Comunicaciones por Satélite en Carmona y la Central Internacional y Telepuerto en Pineda- representan un hito histórico en la evolución de las telecomunicaciones ya no sólo en Andalucía sino también en España que ha tenerse presente en tanto han supuesto un paso decisivo para la mejor integración de la CC.AA. de Andalucía dentro del sistema de telecomunicación internacional, a la vez que ha permitido incorporación de servicios tecnológicamente avanzados al sistema productivo andaluz.

De esta forma, interesa conocer en detalle la forma en que este sistema de telecomunicaciones avanzado vertebra el territorio regional articulando ejes de integración y/o comunicación a nivel internacional, nacional, regional e intrarregional.

FIGURA 2

ESTRUCTURA FUNCIONAL DEL SISTEMA DE TELEFONÍA RURAL MEDIANTE ACCESO CELULAR

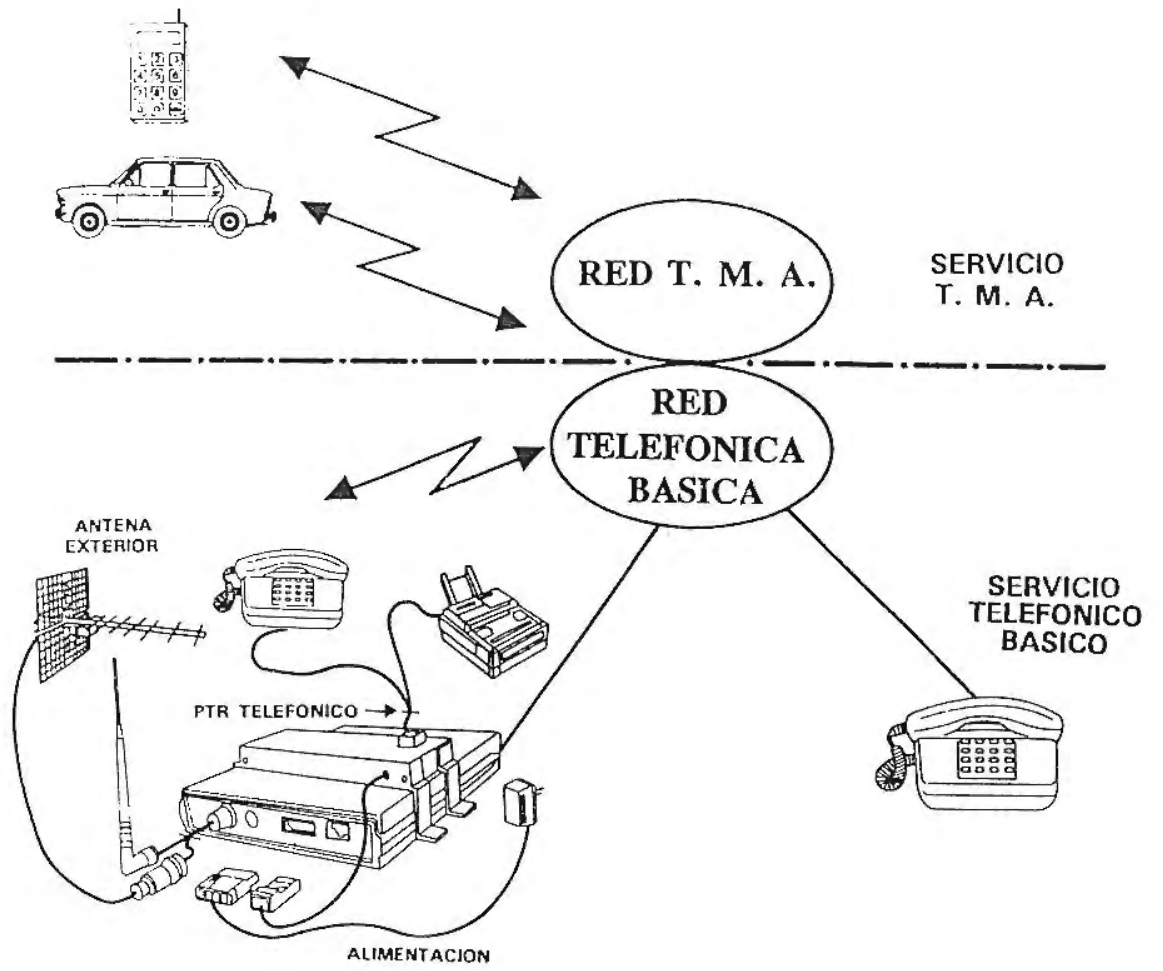

Fuente: C. García, 1992. 


\subsection{Escalas de integración que establece la configuración del actual sistem: de telecomunicaciones en Andalucía}

Es evidente que la articulación del sistema de telecomunicaciones a escal regional esboza en la práctica el modelo de ordenación territorial que establece 1. política territorial de la CC.AA. Andaluza, al integrarse en este caso con la pautas mismas de la política sectorial de telecomunicaciones del Ministerio di Obras Públicas y Transportes.

Es por esto que el sistema se articula jerárquicamente en base la operatividar de centros y redes con diferentes niveles de cobertura, que diseñan diversa escalas de integración para Andalucía.

A este respecto, conviene indicar cómo el proceso de implantación de est moderno sistema en Andalucía ha sido planteado a nivel estatal, ante la máximi de articular y garantizar la operatividad del sistema a nivel nacional, hecho er ocasiones a incidido en la puesta en marcha de actuaciones que apoyan en intere ses estratégicos externos y que como puede intuirse esboza en ocasiones li pervivencia del antiguo modelo territorial centralista, en base al cual la red dt telecomunicaciones adopta un dispositivo radial, que ahora sí como se verá en e caso de la fibra óptica apoya en centros subregionales a partir de los cuale: distribuyen las comunicaciones que canaliza.

Es por esto que a escala internacional la funcionalidad del sistema de telecomuni. caciones en Andalucía permitirá sin lugar dudas la incorporación de la región a contexto tanto europeo como internacional tal y como garantiza, por un lado, li distribución cables submarinos al posibilitar además de la actual conexión con la rec EE.UU.-Países Centroeuropeos, la ampliación de las conexiones con el resto de la fachada mediterránea, Norte de África y Aménica Latina (véase figura 3).

Lo mismo puede decirse de la fibra óptica cuya cobertura valorada a est‘ misma escala además de potenciar las interrelaciones a nivel nacional, se consti. tuye en soporte base para las comunicaciones internacionales hacia el Atlántico fachada Mediterránea y, especialmente, y, resto de Europa.

Sin embargo, especial importancia ha tenido en el contexto de la CC.AA de Andalucía la operatividad de los complejos de comunicaciones avanzadas de Carmona y Pineda -ambos localizados en Sevilla-, los cuales conectados a redes internacionales a través de varias vías garantizan la transmisión de todo tipo de señales de voz, datos e imágenes, estando hoy por hoy considerados como los centros mejor dotados de su género de España ${ }^{4}$.

4. Andalucía Económica, 1992 y Stella Benot, 1994. 
FIGURA 3

DISTRIBUCIÓN DE LAS INFRAESTRUCTURAS DE TELECOMUNICACIONES EN

ANDALUCÍA: SU INTEGRACIÓN A ESCALA INTERNACIONAL

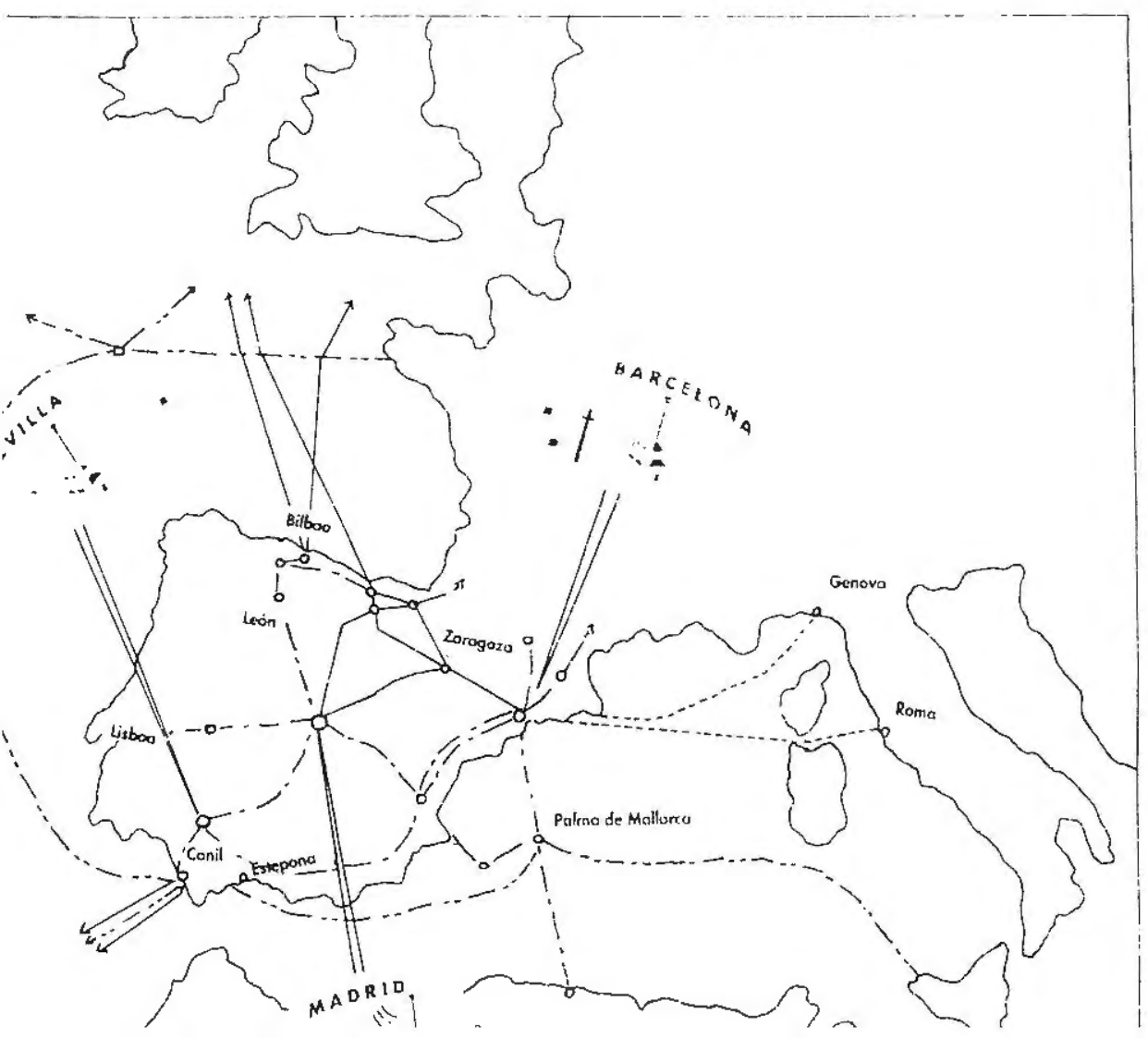

INFRAESTRUCTURA DE TELECOMUNICACIONES

CABLE OPIKO $13 \mu \mathrm{m}$

CABLE OPTICO 15 HM

RADIO ENIACE DIGITAL

SISTEMA DKGITAL CDAXIAL

SISIEMA ANALOGICO COAXIAI

- central

ANAIOGICA

INTERNACIONA - CENTRAL DIGITAL

$\lceil$ torre de telecomunicacion

1 
El primero de ellos, el Centro de Comunicaciones por Satélite, ubicado c escasos kilómetros de Carmona (Sevilla) atiende el tráfico internacional de telefonía, telegrafía, datos, servicios empresariales asociados al área internacional d $\epsilon$ Andalucía, a lo que se suma el tráfico de intercambio de señales de televisión cor origen y destino en esta zona, mediante la utilización de los satélites de las organizaciones EUTELSAT (ámbito europeo) e INTELSAT (ámbito mundial).

Éste se conecta con el de Pineda a través de dos sistemas de enlace con rutas de acceso físicamente distintas, disponiendo actualmente de cuatro estaciones en tierra, dos de ellas de altas prestaciones -Sevilla 1 y Sevilla 2- que cursan el tráfico internacional y de televisión a través de su conexión con los satélites mencionados. Las estaciones emplean técnicas digitales con excepción de las señales de televisión, que utilizan técnicas de modulación analógicas.

La instalación Sevilla 1 cubre el área de Europa mediante acceso a EUTELSAT, siendo la tercera estación de estas características instaladas en España, junto con Guadalajara 1 y Aguimes 2, esta última en Canarias. Está equipada para transmitir y recibir dos señales de televisión (PAL y NSTC) con tres audios simultáneamente.

Por su parte, Sevilla 2 opera dentro de la red del INTELSAT para cubrir las conexiones hacia el área del Atlántico y Oriente Medio, siendo posible la ampliación de su cobertura territorial en un futuro. Por su parte, las estaciones Sevilla 3 y 4 operan cursando servicios empresariales tales como enlaces digitales para empresas, videoconferencias y oferta de redes de datos vía satélite utilizando para ello los sistemas EUTELSAT e INTELSAT Business Services.

Además, en el centro de Carmona también se prevé la instalación de estaciones para enlaces mediante el Sistema de Comunicaciones por Satélites INMARSAT, que cursa tráfico móvil marítimo y aeronaútico vía satélite.

Junto a estas estaciones, tienen gran importancia los cables submarinos que tradicionalmente ha garantizado la conexión de la región con otros países del Mundo a través de los dos centros de Conil (Cádiz), y Estepona (Málaga). Pese a todo, con los avances tecnológicos Telefónica ha optado en los últimos años por mantenerlos y adaptarlos a los nuevos tiempos sustituyendo los antiguos cables por la fibra óptica.

Complementaria a la operatividad del Centro de Comunicaciones por Satélite de Carmona resulta el Centro de Comunicaciones Internacionales de Pineda (Sevilla), el cual integrado en la red de comunicaciones nacional completará progresivamente su estructura digital para atender las necesidades de comunicaciones internacionales españolas hasta finales de los 90 .

En él operan cuatro unidades: una central automática, un centro internacional de transmisión, el telepuerto y el centro IBERMIC dotadas de la más moderna 
tecnología y con capacidad para soportar todo tipo de servicios. La central automática internacional con 12.800 enlaces posee capacidad para tratar unas 75.000 comunicaciones internacionales por hora, pudiendo a su vez conectarse con cualquier central del mundo por facilidades terretres submarinas y vía satélite. A escala nacional su servicio atiende a Andalucía, Extremadura y Canarias.

Por su parte, el centro internacional de transmisión de Pineda se constituye en el punto donde confluyen los diversos medios de enlace de fibra óptica que conectan este centro con las redes nacionales y exteriores, mientras el telepuerto -instalación orientada al sector negocios cuyo objetivo fundamental es facilitar soporte inmediato vía satélite a cualquier demanda de ciruitos digitales de carácter empresarial-, se halla equipado con dos estaciones terrenas orientadas al EUTELSAT.

También, el Centro IBERMIC Internacional instalado en el Centro de Comunicaciones Internacionales de Pineda, es uno de los cuatro centros previstos en la red internacional española para la conexión de la red IBERMIC con centros de gestión y operación equivalentes en el extranjero. Su objetivo no es otro sino garantizar las dotaciones de facilidades técnicas y operativas para la inmediata constitución y posterior mantenimiento, de circuitos digitales internacionales de cualquier velocidad de uso privado.

En base, por tanto, al esquema trazado para el sistema de telecomunicaciones a escala internacional es necesario resaltar la función de Sevilla como centro regional o «de primer orden» en el contexto de la CC.AA. de Andalucía, a partir del cual se canalizan y distribuyen las comunicaciones. Desde esta perspectiva, emerge sin competencia alguna la potencialidad funcional de Sevilla de cara al mantenimiento de los niveles de operatividad del sistema de telecomunicaciones en Andalucía. Pese a todo, como se decía anteriormente la concentración de la infraestructura de telecomunicaciones en este caso no interpone límites a la comunicaciones de otros centros y, en definitiva, ámbitos que quedan bajo la cobertura de éstos, en base a la distribución por territorio regional de cables y sistemas de transmisión tal y como puede verse sobre la misma figura 4, cuya operatividad, cn última instancia, garantiza las comunicaciones del conjunto del territorio andaluz hacia el exterior.

Se entiende, por tanto, como a escala internacional el actual sistema de telecomunicaciones en Andalucía se articula reproduciendo el esquema diseñado por el modelo teritorial andaluz. Un esquema de integración que a nivel intrarregional hace converger las comunicaciones desde los distintos centros subregionales hacia el Sevilla, centro regional de primer orden como se decía dentro del sistema de telecomunicaciones de la CC.AA. de Andalucía. 
Paralelamente, a nivel estatal, puede decirse es la progresiva cobertura del territorio mediante fibra óptica la que garantiza la integración de las diferentes Comunidades Autónomas. Así la progresiva introducción de la fibra óptica en Andalucía he conllevado la conexión de las capitales provinciales y de los centros subregionales de Jerez y Algeciras, integrándose dicha red con la de las Comunidades Autónomas limítrofes a través de los grandes ejes comunicación que enlazan éstas con las capitales de provincia más próximas (véase figura 3).

Para ello, cada uno de estos centros se están dotando, según lo previsto, de un centro provincial de servicios integrados de telecomunicación, si bien de segundo orden en el contexto regional, desde donde se difundirán al resto de cada provincia los nuevos servicios. Con estas intervenciones sectoriales, por tanto, debe entenderse se están tratando de paliar la desestructuración del sistema urbano andaluz y, en consecuencia, de su territorio, al contribuir decididamente a la mejora de la base económica regional, dado que en este caso el desarrollo del sistema de telecomunicaciones parece plantearse a partir de la integración intrarregional del espacio andaluz.

Pese a todo, cabe pensar cómo la falta de estructuración a escala regional del sistema urbano y la debilidad y desigualdades a nivel intrarregional de las condiciones de desarrollo imponen limitaciones de base, hoy por hoy, al desarrollo efectivo de este modemo sistema de telecomunicaciones, dado que la utilización del mismo por parte de la población andaluza, por el momento, resulta ser limitada.

De cualquier modo, este sistema de telecomunicaciones en Andalucía ha sido propuesto en tanto, por un lado, cohesiona y refuerza la estructura productiva y la base empresarial regional y, por otro, eleva el rango funcional de la región y/o ciudades que se dotan del mismo, abriendo así posibilidades para la configuración de la región frente al exterior y, en último término, para la mejor articulación del territorio andaluz.

\section{CONCLUSIÓN}

Ciertamente, como puede entenderse a partir de lo expuesto los esfuerzos actuales pretenden dotar a la región de una infraestructura avanzada de telecomunicaciones dado que contribuirán a cambiar la imagen de la región y a reforzar su potencialidad real en el exterior.

Y es que la globalización económica actual implica que cada vez territorios más distantes se encuentren integrados funcionalmente, pero también que ese proceso de descentralización económica que lleva a integrar territorios cada vez más vastos se acompañe, por exigencias del mismo sistema, de un proceso muy 
FIGURA 4

DISTRIBUCIÓN DE FIBRA ÓPTICA EN ANDALUCÍA: ESTRUCTURA DE LA RED A

NIVEL AUTOMÁTICO Y ESTATAL

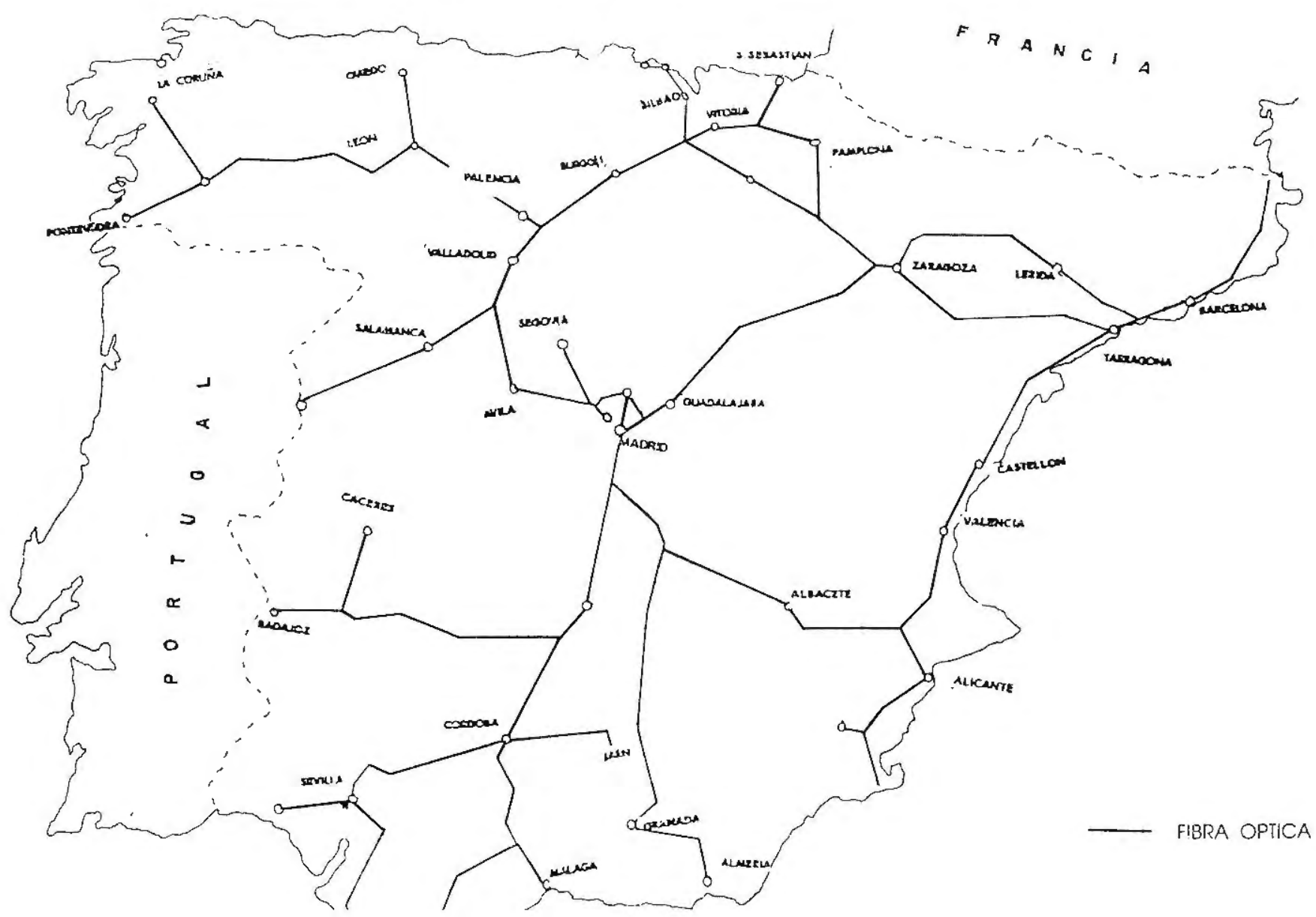


selectivo de concentración espacial de las actividades decisionales de alto nive entomo a las ciudades o metrópolis, en tanto nodos desde los que se organiza li producción a nivel mundial, se articulan los mercados, se generan nuevas tecno logías,...

Ello se observa en la estructura misma que se observó define el sistema d telecomunicaciones andaluz donde claramente emerge el papel de Sevilla, comc nodo central desde el que se canalizan las comunicaciones tanto hacia el exterio] como hacia el resto de la Comunidad Autónoma, dado que concentra los equipos de control, gestión y mantenimiento de la red de telecomunicaciones a nive regional.

Sin embargo, puede decirse que la articulación territorial del sistema de telecomunicaciones en Andalucía parece imponer de hecho pautas para la integración económica del territorio sobre el que se proyecta, que para nada resulta aleatoria sino resultado directo de intervenciones tendentes a primar una determinada forma de organización y/u ordenación de la actividad económica regional que apoya y se traduce, finalmente, sobre el sisterna de ciudades que se observó se configuraba a partir de su articulación en base a la conexión telemática de Sevilla con las capitales provinciales -Huelva, Córdoba, Cádiz, Málaga, Granada, Jaén y Almería- y los centros subregionales de Algeciras y Jerez, reforzándose así, en primer lugar, el papel central de la capital en el contexto regional andaluz, y, en segundo, de las capitales provinciales como centros subregionales de segundo orden.

Sin embargo, debajo de todo este plantel inicial se observa una problemática más amplia en el sentido de que hoy cualquier ámbito territorial que quede fuera de este sistema infraestructural y de relaciones queda realmente postergado en su desarrollo, lo que hace a juicio personal sobresaltar la necesidad de dirigir el desarrollo del sector primando no sólo intervenciones de tipo sectorial, sino también aunando intimamente a éstas una dimensión territorial, dado que la fuerte concentración de infraestructuras y oferta de servicios telemáticos avanzados en determinados zonas como resulta ser el caso en la práctica de Sevilla o, puntualmente, sobre los centros subregionales antes mencionados no hace más que incrementar las ineficiencias y las desigualdades intrarregionales, por la desigualdad de oportunidades económicas que genera un mapa con estas características.

Cierto es que, difícilmente, podría canalizarse uniformemente proyectos para el desarrollo de las telecomunicaciones en la región, pero, de cualquier forma, lo que tampoco conviene es subestimar la potencialidad de ámbitos territoriales más débiles como son los municipios serranos andaluces, siendo oportuno en este caso específicamente el desarrollo de una correcta intervención pública que se 
adecúe y sirva de estímulo al sector privado, y sea base para el desenvolvimiento de la actividad económica.

Un elemento adicional parece completar, finalmente, este discurso en el sentido que tal y como ha quedado recogido en este estudio resulta evidente la incidencia que sobre el sector está teniendo la actual política desreguladora, que se impone a nivel internacional al provocar una transformación radical sobre todo por lo que afecta al control del mercado de los servicios de telecomunicaciones, en tanto abiertos progresivamente a la libre concurrencia y que adquieren día a día sobre todo aquellos de alto valor añadido una rentabilidad elevada, que se acentúa imperativamente si se observa por relación con la oferta de servicios telemáticos avanzados.

Desde esta óptica, la intervención pública debería cohesionar la oferta/demanda de tales servicios a través de la promoción y desarrollo de nuevas empresas de telecomunicaciones en Andalucía. Ahora bien, no debe de perderse de vista que estas empresas encuentran hoy un mercado preferente en las mismas empresas regionales y no en los usuarios privados lo que de forma lógica implicaría que se valorasen los requerimientos de las mismas antes de estimular una oferta de servicios telemáticos avanzados, que para nada se adapte a las exigencias del tejido productivo andaluz.

Tratando, por tanto, de concretar todas estas argumentaciones que subyacen al desarrollo de este estudio como ha podido ir valorándose conviene finalmente observar en consecuencia en qué se traducirán todas estas circunstancias por lo que afecta al desarrollo futuro del sector de las telecomunicaciones en Andalucía y es por ello que no cabe más que manifestar nuevamente la incertidumbre y, hasta cierto punto, el excepticismo que se cierne entomo a estas circunstancias en tanto las características del sistema económico y la estructura actual del sector de las telecomunicaciones difícilmente si se estima de forma objetiva permitirá configurar un sector dinámico con potencialidad intrínseca para revitalizar la realidad económica y territorial andaluza.

De cualquier forma, no sería justo minusvalorar los esfuerzos actuales que se vienen realizando en la CC.AA. de Andalucía dado que a corto plazo las iniciativas tendentes al desarrollo de las telecomunicaciones en la región contribuirán en cierta medida a favorecer la integración si no de la región sí de algunos núcleos de la misma en la red de ciudades europeas y en el sistema económico internacional a través del desarrollo progresivo del modemo sistema de telecomunicaciones que se describía, capaz en último término de favorecer el desarrollo económico mismo de la región.

Emerge, por tanto, en último término, el papel de las nuevas tecnologías de telecomunicaciones en Andalucía como elemento de primer orden llamado a 
favorecer ese salto tecnológico, empresarial y de mercado que hoy trata de dar la región para incorporarse como una región moderna dotada de nuevas funciones al escenario tanto estatal, como europeo e internacional, abriendo paralelamente nuevas posibilidades para la misma.

\section{BIBLIOGRAFÍA}

Andalucía económica. «El presupuesto andaluz para 1994». Pp.38-43. №41. Enero, 1994.

Andalucía económica. «Sevilla comunica al mundo». Sección Telecomunicaciones. №19. Enero, 1992.

Benot, S.: «Sevilla en centro de tecnología punta en telecomunicaciones». En Diario ABC. Pp.56-57. Miércoles, 25-5-1994.

Consejería de Política Territorial: El sistema de ciudades. Junta de Andalucía, 1985.

Consejería de Política Territorial: Bases para la Ordenación del Territorio en Andalucía. Junta de Andalucía, 1990.

Esteve Secall,R.: Población, actividad económica y territorio. En M. Martín Rodriguez (Direct.). Pp.555-582. Estructura económica de Andalucía. Biblioteca de Economía. Serie Manuales. Ed.Espasa Calpe, 1993.

Fajardo, P.: «Línea con el mañana». En Rev. MOPT. Pp. 115-119. Mayo, 1992. Feria Toribio, J.Mª: El sistema urbano andaluz. En G.Cano García (Coord.).

Geografía de Andalucía. Tomo III. Pp. 259-345. Ed. Tartessos. 1987.

García Gómez, C.: «La distancia no es el olvido». En Rev. MOPT. Pp.53.57. Diciembre, 1992.

Grupo PINTA: El desafío andaluz. Modemización tecnológica y desarrollo económico en Andalucía. IFA y Expo'92. Sevilla, 1990.

Jordá Borrell,R.: Población, Economía y Territorio en Andalucía. En G.Cano

García (Coord.). Geografía de Andalucía. Tomo III. Pp. 137-206. Ed.

Tartessos. 1987.

Jordá Borrell, R.: «Transformaciones recientes de la economía andaluza». En Rev. Estudios Andaluces, nº16, 1991. Pp. 46-58.

Jordá Borrell, R.: Las relaciones Ciencia-Tecnología-Industria andaluza y el papel de la Administración. Instituto de Desarrollo Regional. Sevilla, 1992.

Ocaña Ocaña, $\mathbf{M}^{\mathbf{a}}$ del C.: Ordenación del territorio e infraestructuras. En M. Martín Rodriguez (Direct.). Pp.147-184. Estructura económica de Andalucía. Biblioteca de Economía. Serie Manuales. Ed.Espasa Calpe, 1993. 
Resolución 16/6/1993 del Consejo relativa a al informe sobre la situación del sector de las telecomunicaciones. Bol. CE 6-93.

Velázquez, C.: Servicios avanzados para las empresas. En Rev. Andalucía económica. Sección Telecomunicaciones. №19. Enero, 1992. 